\title{
Crystalline Characterization of TlBr Semiconductor Detectors Using Wavelength-resolved Neutron Imaging
}

\author{
Kenichi Watanabe, ${ }^{1 *}$ Kio Matsumoto, ${ }^{1}$ Akira Unitani, ${ }^{1}$ \\ Keitaro Hitomi, ${ }^{2}$ Mitsuhiro Nogami, ${ }^{2}$ and Winfried Kockelmann ${ }^{3}$ \\ ${ }^{1}$ Department of Applied Energy Engineering, Nagoya University, Furo-cho, Chikusa-ku, Nagoya 464-8603, Japan \\ ${ }^{2}$ Department of Quantum Science and Energy Engineering, Tohoku University, Sendai 980-8579, Japan \\ ${ }^{3}$ STFC, Rutherford Appleton Laboratory, ISIS Facility, Harwell, OX11 0QX, United Kingdom
}

(Received December 19, 2019; accepted March 10, 2020)

Keywords: TlBr semiconductor detector, neutron diffraction, energy-resolved neutron imaging

Thallium bromide ( $\mathrm{TlBr}$ ) has been one of the promising gamma-ray detector materials because of its high gamma-ray attenuation length, availability of room-temperature operation, and relatively high energy resolution. However, the high-resolution detector has been limited to a relatively small size. Hence, the next task in the $\mathrm{TlBr}$ development is to establish fabrication processes of large detectors. As one of the candidates for evaluating the TlBr crystal quality, we demonstrated neutron Bragg-dip imaging, which is one of the neutron diffraction techniques and is based on wavelength-resolved neutron imaging. All the studied samples seemed to be imperfect crystals and slightly distorted. Although differences in crystal orientation distributions among the samples cannot be obtained from the present data, it is suggested that one of the crystals has a boundary, or possibly a small crystal grain near the crystal center, which may result in a low-quality electron mobility and lifetime $(\mu \tau)$ product.

\section{Introduction}

Thallium bromide $(\mathrm{TlBr})$ is one of the promising candidates as a high-energy-resolution compound for semiconductor gamma-ray detectors. ${ }^{(1-3)}$ Since this material is made from high-atomic-number elements $(\mathrm{Tl}=81, \mathrm{Br}=35)$ and has a high density $\left(7.56 \mathrm{~g} / \mathrm{cm}^{3}\right)$, the $\mathrm{TlBr}$ detector has an advantage in detection efficiency because of its small attenuation length for gamma rays. The band-gap energy of $\mathrm{TlBr}$ of $2.68 \mathrm{eV}$ is higher than those of germanium $(0.67 \mathrm{eV})$ and silicon $(1.11 \mathrm{eV})$, resulting in a high crystal resistivity and its suitability for roomtemperature operation. From the viewpoint of the fabrication process of the detector, a crystal can be grown directly from the melt, because it can melt congruently at $460{ }^{\circ} \mathrm{C}$ and exhibits no destructive phase transition below the melting point. These promising physical properties have promoted the development of the TlBr gamma-ray detector.

In this decade, the purification process of $\mathrm{TlBr}$, such as zone purification, has been improved considerably. ${ }^{(4)}$ Therefore, the mobility-lifetime products of electrons and holes of TlBr have

*Corresponding author: e-mail: k-watanabe@energy.nagoya-u.ac.jp

https://doi.org/10.18494/SAM.2020.2744 
been improved. This means that charge carriers can easily be collected. Although the mobility of holes is much lower than that of electrons, this problem was overcome by applying a single polarity charge sensing technique, such as the use of a capacitive Frisch grid or pixelated detectors. ${ }^{(5,6)}$ Consequently, $\mathrm{TlBr}$ detectors achieved a high energy resolution of roughly $1 \%$ for $662 \mathrm{keV}$ gamma rays. ${ }^{(5)}$ However, these results were obtained from relatively small crystal detectors with sizes of $5 \mathrm{~mm}$ or less. The next step of $\mathrm{TlBr}$ development is to increase the detector size or detection efficiency. The current key issue is how we can control the quality of the fabrication processes to realize a large-volume detector. Although these fabrication processes take a long time, such as crystal purification, growth, dicing, polishing, forming electrodes, and wiring cables, we cannot evaluate the detector performance or crystal quality before completing the fabrication processes. One of the most important material features affecting the detector performance is the quality of the $\mathrm{TlBr}$ crystal. It is therefore of interest to evaluate the crystal quality separately to identify problems in the fabrication processes. Since $\mathrm{TlBr}$ is a relatively plastic or soft material, it might easily be distorted not only during crystal growth but also during the ensuing fabrication processes such dicing and polishing. The fabrication processes of $\mathrm{TlBr}$ detectors might markedly be improved if a crystal evaluation method will be established. An energy-resolved neutron imaging technique is a promising candidate for evaluating the crystal growth and treatment processes of $\mathrm{TlBr}$. In particular, Bragg-dip imaging for single-crystal samples, such as Bragg-edge imaging for polycrystalline samples, can easily acquire two-dimensional crystal information. ${ }^{(7,8)}$

\section{Materials and Methods}

\subsection{Sample preparations}

We prepared a series of $\mathrm{TlBr}$ single-crystal samples, which have various impurity levels or were taken from different positions in the crystal ingot. As a crystal growth starting material, $\mathrm{TlBr}$ beads of $99.999 \%$ purity were used. The raw material and hydrogen bromide gas were sealed in a quartz ampule. The hydrogen bromide gas was used to remove oxide and hydroxide impurities from the $\mathrm{TlBr}$ melt and to suppress the vaporization of TlBr. The raw material was further purified by using a horizontal zone purification system with two resistive heaters, which were moved at a speed of $50 \mathrm{~mm} / \mathrm{h}$. The process was repeated 114 times. This procedure was determined on the basis of a previous study. ${ }^{(4)}$ Finally, a TlBr crystal was grown by a single zone pass with heaters moving at a speed of $1 \mathrm{~mm} / \mathrm{h}$. The grown $\mathrm{TlBr}$ crystal is shown in Fig. 1(a). From the upstream and downstream regions of the zone purification, four samples each were diced into a size of roughly $5 \times 5 \times 1.8 \mathrm{~mm}^{3}$. All surfaces were then polished. The upstream samples are considered to have a higher purity than the downstream ones. Before neutron imaging, electron mobility and lifetime $(\mu \tau)$ products were measured as for previous detector evaluations. To evaluate the $\mu \tau$ products, gold electrodes were deposited on the diced crystals by vacuum evaporation. Figure 1(b) shows the fabricated detector.

The $\mu \tau$ products of electrons in the fabricated detectors were determined by Hecht's method. ${ }^{(9)}$ For Hecht's method, each $\mu \tau$ product is determined by using the difference in charge collection 


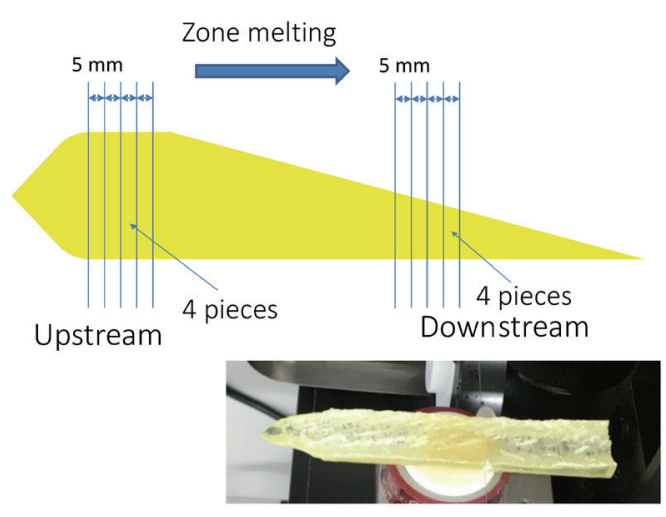

(a)
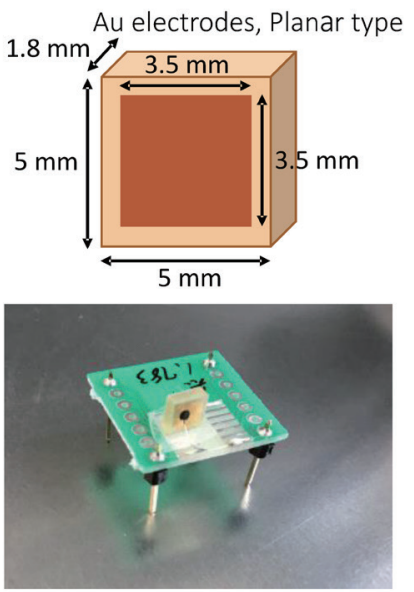

(b)

Fig. 1. (Color online) Prepared samples. (a) Sampling positions from the TlBr crystal ingot. (b) Detector geometry for previous detector evaluation before neutron imaging.

with various bias voltages. The cathode surface of the detector was irradiated with ${ }^{241} \mathrm{Am}$ gamma rays, whose energy was $59.5 \mathrm{keV}$ and low enough to be absorbed just behind the cathode surface. The electrons created by $59.5 \mathrm{keV}$ gamma rays traversed almost the entire detector thickness. The pulse height spectra were measured while varying the applied bias voltage to the detector. In this experiment, the applied voltage was changed from 10 to $100 \mathrm{~V}$. For these crystals, when applying more than $200 \mathrm{~V}$, the leak current significantly increased. Peak positions in the spectra were determined as a function of the applied voltage. The $\mu \tau$ product of electrons can be derived from the following equation: ${ }^{(9)}$

$$
N=N_{0} \exp \left(-\frac{t}{\tau}\right)=N_{0} \exp \left(-\frac{1}{\tau} \frac{D}{\mu(V / D)}\right)
$$

where $N$ stands for the peak position at the applied voltage $V, N_{0}$ is also the peak position when electrons are completely collected, $D$ is the detector thickness, and $\mu$ and $\tau$ are the electron mobility and lifetime, respectively. The experimental data of the applied voltage dependence of the peak positions were fitted to this equation to determine the $\mu \tau$ product.

\subsection{Energy-resolved neutron imaging}

We acquired energy-resolved neutron images at the IMAT beamline at the pulsed neutron facility ISIS, UK. ${ }^{(10,11)}$ In this experiment, the neutron flux was roughly $5 \times 10^{6} \mathrm{n} / \mathrm{cm}^{2} / \mathrm{s}$. The neutron wavelength can be determined by the neutron time-of-flight method using a timeresolving detector. One of the neutron applications to obtain crystal structure information is the neutron diffraction method. A neutron Bragg-edge imaging technique combines neutron transmission imaging with neutron diffraction to extract crystalline information 
from polycrystalline materials. For single crystal analysis, a Bragg-dip pattern can be observed instead of the Bragg-edge pattern. Figure 2 shows a conceptual drawing of the neutron Bragg-dip imaging technique. From wavelength-resolved transmission images, the neutron transmission spectra at all pixels can be extracted. For a single crystalline sample, the transmission spectra exhibit a dip structure at the neutron wavelength, where neutrons are diffracted out of the incident beam direction. A pattern of these dip positions gives us information about the crystal orientation. In this paper, we apply the neutron Bragg-dip imaging technique to evaluate the crystalline quality of the TlBr single crystal.

As a time-resolved two-dimensional neutron detector, we applied the neutron-sensitive microchannel plate $(\mathrm{MCP}) /$ Timepix detector. ${ }^{(12,13)}$ The detector has $512 \times 512$ pixels with a size of $55 \mu \mathrm{m}$. In the TOF experiments, the Timepix was operated in the event timing mode, in which the time of arrival of individual neutron signals relative to the trigger signals of neutron pulses was recorded in each pixel. The FPGA-based data processing unit creates threedimensional histograms, with two dimensions in space and one dimension in neutron flight time. The neutron flight path length was $56.4 \mathrm{~m}$. Figure 3 shows the neutron-sensitive MCP

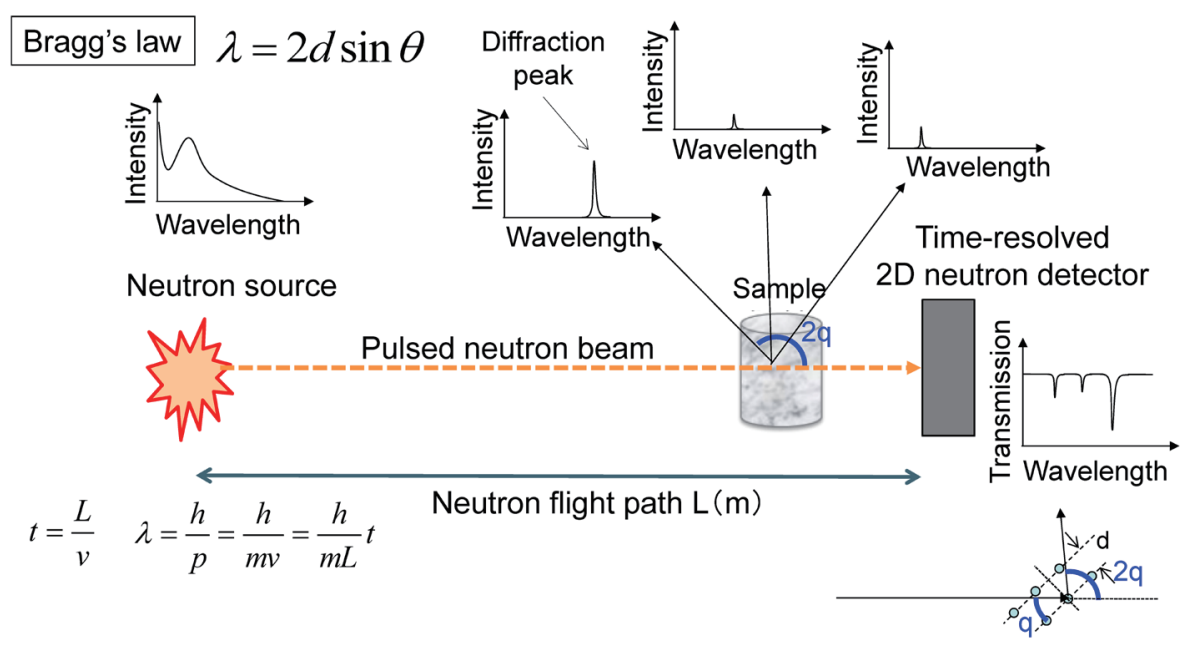

Fig. 2. (Color online) Conceptual drawing of the neutron Bragg-dip imaging technique.

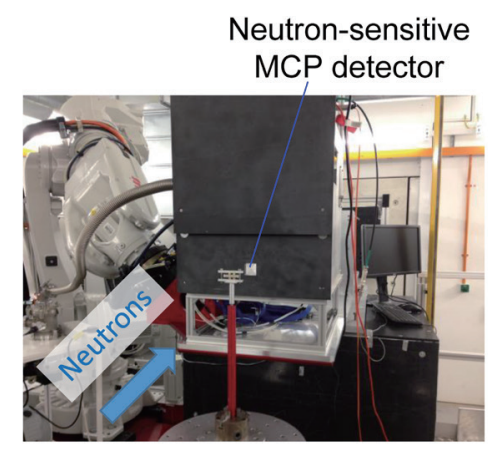

(a)

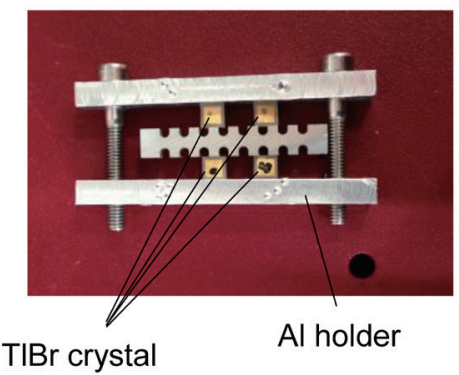

(b)

Fig. 3. (Color online) Photographs of (a) neutron-sensitive MCP detector and (b) TlBr samples. 
detector and $\mathrm{TlBr}$ sample mount. The data collection time for samples and the open beam was $8 \mathrm{~h}$ each for a neutron wavelength range from 0.2 to $0.6 \mathrm{~nm}$. The L/D was 125 , enabling a spatial resolution of about $0.24 \mathrm{~mm}$.

\section{Results and Discussion}

\section{$3.1 \mu \tau$ products}

For the upstream and downstream samples, the $\mu \tau$ products were determined by fitting the applied voltage dependence of the peak positions to Hecht's equation. Figure 4 shows the $\mu \tau$ products obtained from the eight prepared samples. Unfortunately, although a clear difference in $\mu \tau$ products between the upstream and downstream samples cannot be observed, some downstream detectors show relatively low $\mu \tau$ products compared with others.

\subsection{Energy-resolved neutron imaging}

Figure 5 shows basic 'white-beam' neutron radiographic images obtained from the prepared $\mathrm{TlBr}$ samples, showing the $\mathrm{TlBr}$ crystals and aluminum holders. Conductive carbon paste, which was used for wire connection, can also be seen in these images. However, we cannot extract any information about the $\mathrm{TlBr}$ crystals from these images, which average the neutron transmission over the whole wavelength region. Figure 6 shows energy- or wavelength-resolved neutron transmission images. When changing the neutron wavelength, black regions are gradually moving in the T1Br crystals. The black color stands for low neutron transmission or high attenuation. When the neutron wavelength matches Bragg's diffraction condition, neutrons are diffracted out and cannot arrive in the detector. This means that a location matching Bragg's law shows black color. For a perfect crystal, with the crystal lattice perfectly aligned across the full size of the crystal, the entire region of the crystal simultaneously becomes black at the neutron wavelength satisfying Bragg's diffraction law. Therefore, these images indicate that the prepared $\mathrm{T} 1 \mathrm{Br}$ samples are either perfect or imperfect, and slight distortions

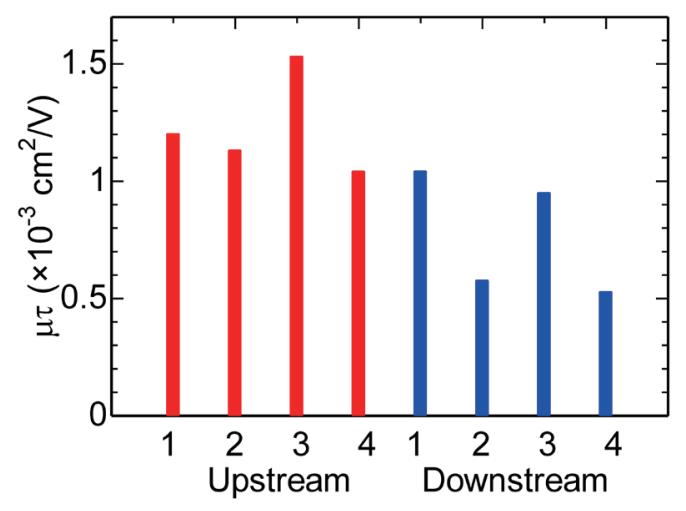

Fig. 4. (Color online) $\mu \tau$ products obtained from prepared samples. 


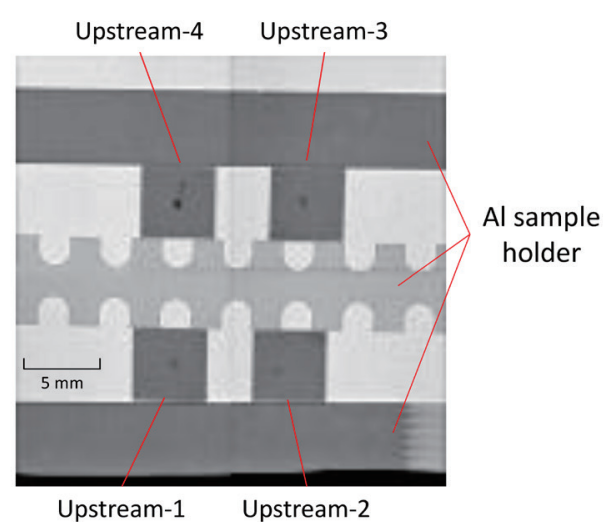

(a)

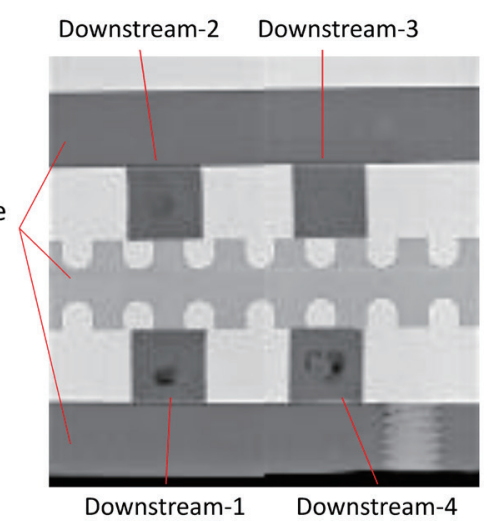

(b)

Fig. 5. (Color online) Simple radiographic images of the (a) upstream and (b) downstream samples. These images are averaged over the entire wavelength range.

a) $\lambda=0.335 \mathrm{~nm}$

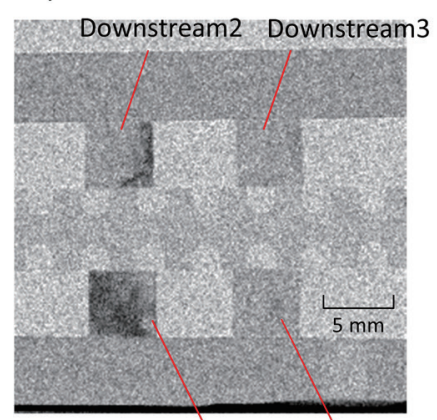

Downstream1 Downstream4

(a) b) $\lambda=0.344 \mathrm{~nm}$

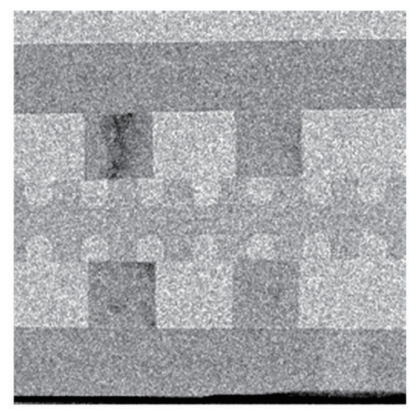

(b) c) $\lambda=0.355 \mathrm{~nm}$

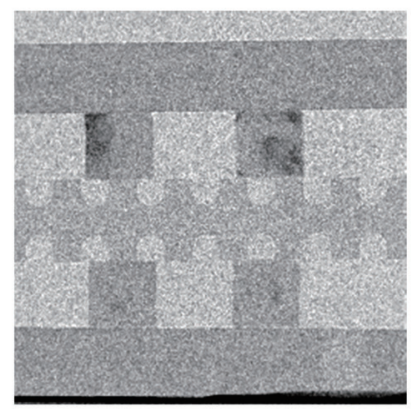

(c)

Fig. 6. (Color online) Wavelength-resolved neutron transmission images at the neutron wavelengths $\lambda$ of (a) 0.335 , (b) 0.344 , and (c) $0.355 \mathrm{~nm}$.

become evident. Figure 7 shows examples of neutron transmission spectra obtained at different positions in the same $\mathrm{TlBr}$ crystal. The dip pattern slightly shifted depending on the position in the crystal. This also indicates that the crystal orientation is gradually changing in the crystal.

\subsection{Neutron Bragg-dip imaging}

The space group of T1Br is Pm-3m (\#221) and the lattice parameter is $a=3.959 \AA .^{(14)}$ Hence, the relative orientation angles of the crystal to the neutron beam can be determined from the Bragg-dip positions as shown in Fig. 8(a). We conducted a Bragg-dip fitting procedure to identify the $\mathrm{TlBr}$ crystal orientations. Figure 8(b) shows an example of a spectrum fitted to the experimentally obtained transmission spectrum. In this fitting curve, the dip position 


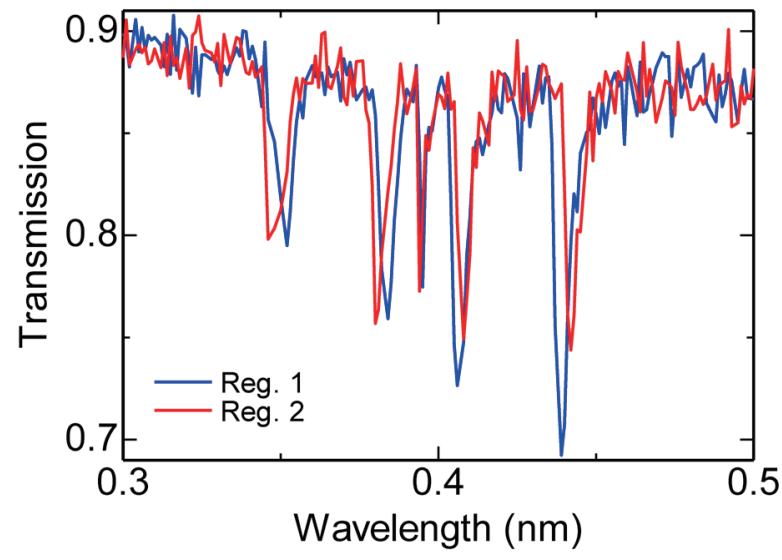

(a)

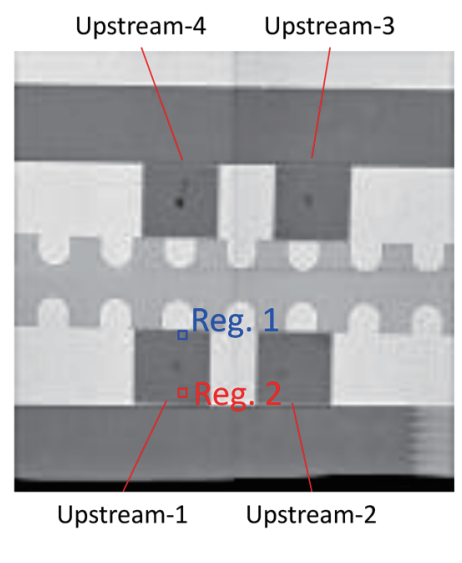

(b)

Fig. 7. (Color online) (a) Neutron transmission spectra in regions 1 and 2 indicated in (b).

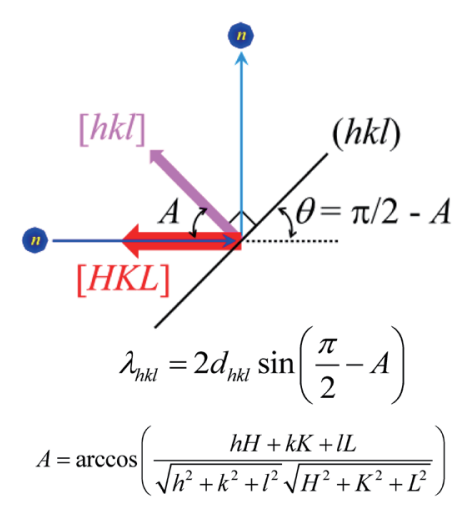

(a)

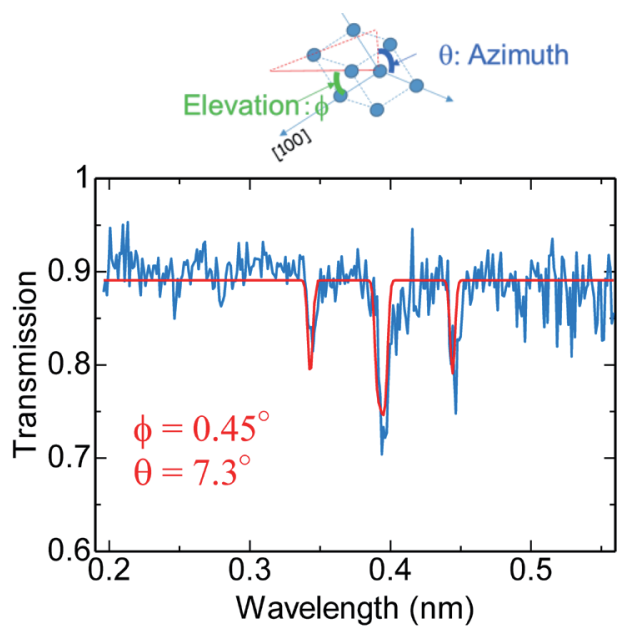

(b)

Fig. 8. (Color online) (a) Directional relationship between a neutron beam axis and a crystal lattice plane. (b) Example of a fitted spectrum to the experimentally obtained transmission spectrum.

is determined by the $\mathrm{TlBr}$ crystal structure and crystal orientation, and the dip shape has a Gaussian distribution. As a result, the extracted parameter in this fitting procedure is crystal orientation. The fitted spectrum well explains a dip pattern in the experimentally obtained one.

Figure 9 shows examples of the crystal orientation distribution reconstructed by Bragg-dip fitting. Unfortunately, a clear difference in crystal orientation distribution between the upstream and downstream samples cannot be confirmed. However, in the downstream-2 sample, which showed the worst $\mu \tau$ product for all the samples, a clear crystal boundary near the crystal center can be seen. In addition, a small crystal grain near the crystal center can also be seen. These features might induce an undesired effect on the $\mu \tau$ product. Although bottom corner regions in 
Upstream-3

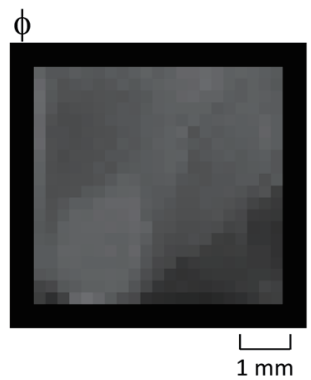

Gray level: 0.0-5.0

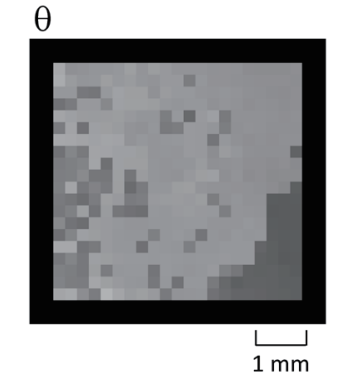

Gray level: 0.0-10.0

(a)

(b)

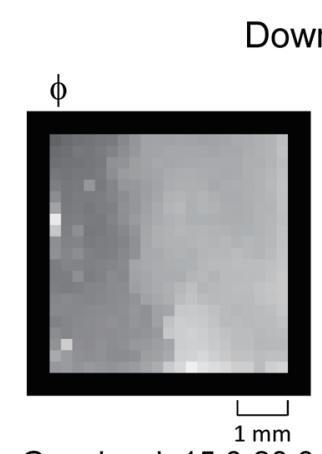

Gray level: 15.0-20.0

(c)
Downstream-2

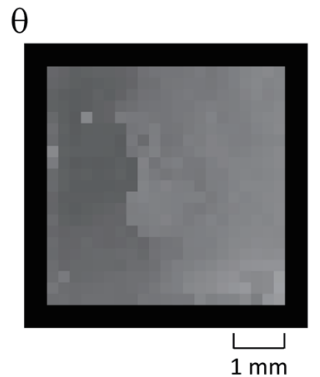

Gray level: 0.0-10.0

(d)

Fig. 9. Examples of the crystal orientation distributions reconstructed by Bragg-dip fitting. (a) Elevation angle $\phi$ distribution in upstream-3 sample. (b) Azimuth angle $\theta$ distribution in upstream-3 sample. (c) $\phi$ distribution in downstream-2 sample. (d) $\theta$ distribution in downstream-2 sample.

the upstream-3 sample also have slight distortion, they might be distorted by the corners of the aluminum holder when fixing the sample for the neutron imaging experiments.

\section{Conclusions}

We demonstrated neutron Bragg-dip imaging, which is one of the neutron diffraction techniques and is based on wavelength-resolved imaging, to evaluate crystal quality or crystal orientation distribution in a $\mathrm{TlBr}$ gamma-ray detector. All the samples seemed to be imperfect crystals, exhibiting slight distortion. Although we compared samples expected to have different impurity levels, unfortunately, a clear difference in crystal orientation distribution among the prepared samples were not confirmed. However, it was suggested that a crystal having a boundary or a small crystal grain near the crystal center might result in a low-quality $\mu \tau$ product. Further investigations are required with samples that display clearly different detector performance characteristics, in order to reveal the relationship between the crystal quality and the detector performance. Ideally, we hope to improve some fabrication processes, such as the dicing and polishing of the crystal, by evaluating the crystal quality by the wavelength-resolved neutron imaging method.

\section{Acknowledgments}

This work was partially supported by the collaboration work with Chubu Electric Power Company Inc. This study was also partially supported by the Cooperative Research Project of the Research Institute of Electronics, Shizuoka University. Experiments at the ISIS Neutron and Muon Source were supported by a beamtime allocation from the Science and Technology Facilities Council DOI10.5286/ISIS.E.RB1900043. 


\section{References}

1 K. Hitomi, T. Murayama, T. Shoji, T. Suehiro, and Y. Hiratate: Nucl. Instrum. Methods Phys. Res., Sect. A 428 (1999) 372.

2 K. Hitomi, T. Shoji, and K. Ishii: J Cryst. Growth 379 (2013) 93.

3 K. Hitomi, T. Onodera, S-Y. Kim, T. Shoji, and K. Ishii: Nucl. Instrum. Methods Phys. Res., Sect. A 747 (2014) 7.

4 K. Hitomi, T. Onodera, and T. Shoji: Nucl. Instrum. Methods Phys. Res., Sect. A 579 (2007) 153.

5 K. Hitomi, T. Tada, T. Onodera, S-Y. Kim, Y. Xu, T. Shoji, and K. Ishii: IEEE Trans. Nucl. Sci. 60 (2013) 1156.

6 K. Hitomi, T. Onodera, T. Shoji, and Z. He: Nucl. Instrum. Methods Phys. Res., Sect. A 578 (2007) 235.

7 H. Sato: J. Imaging 4 (2018) 7.

8 F. Malamud and J. Santisteban: J. Appl. Cryst. 49 (2016) 348.

9 Z. He, G. F. Knoll, and D. K. Wehe: J. Appl. Phys. 84 (1998) 5566.

10 T. Minniti, K. Watanabe, G. Burca, D. E. Pooley, and W. Kockelmann: Nucl. Instrum. Methods Phys. Res., Sect. A $\mathbf{8 8 8}$ (2018) 184.

11 W. Kockelmann, T. Minniti, D.E. Pooley, G. Burca, R. Ramadhan, F. A. Akeroyd, G. D. Howells, C. M. Smith, D. P. Keymer, J. Kelleher, S. Kabra, T. L. Lee, R. Ziesche, A. Reid, G. Vitucci, G. Gorini, D. Micieli, R. G. Agostino, V. Formoso, F. Aliotta, R. Ponterio, S. Trusso, G. Salvato, C. Vasi, F. Grazzi, K. Watanabe, J. W. L. Lee, A. S. Tremsin, J. B. McPhate, D. Nixon, N. Draper, W. Halcrow, and J. Nightingale: J. Imaging 4 (2018) 47.

12 A. S. Tremsin, J. V. Vallerga, J. B. McPhate, and O. H. W. Siegmund: Nucl. Instrum. Methods Phys. Res., Sect. A 787 (2015) 20.

13 K. Watanabe, T. Minniti, W. Kockelmann, R. Dalgliesh, G. Burca, and A. S. Tremsin: Nucl. Instrum. Methods Phys. Res., Sect. A 861 (2017) 55.

14 A. V. Churilov, W. M. Higgins, G. Ciampi, H. Kim, L. J. Cirignano, F. Olschner, and K. S. Shah: Proc. SPIE 7079 (2008) 70790K-1. 